\title{
Computer Simulation Method for the Bubbling Performance of the Vortex Microbubble Generator
}

\author{
Zhekun $\mathrm{Li}^{1, \mathrm{a}}$, Wenli Shi ${ }^{1, \mathrm{~b}}$, Wei He $\mathrm{H}^{1, \mathrm{c}}$, Yujin Fan ${ }^{1, \mathrm{~d}}$ and Hongjun Luo ${ }^{2, \mathrm{e}}$ \\ ${ }^{1}$ School of Mechanical and Electrical Engineering, Kunming University of Science and Technology, \\ Kunming 650500, China \\ ${ }^{2}$ Honghe University, Yunnan Mengzi 661100, China \\ azhekunlikust@sina.com, bswl_ie@163.com, chw_big@126.com, dfanyujinkmust@163.com, \\ e150339309@qq.com
}

Keywords: Computational Fluid Dynamics; Computer Simulation; Bubbling Performance; Vortex Microbubble Generator

Abstract. The vortex microbubble generator relies on the internal strong swirling flow that will make the air from inlet be stirred into tiny bubbles, and then flow mixed with the water from the outlet. Although the structure of the vortex microbubble generator is simple, the sizes of the structure parameters, such as the diameters of inlet and outlet, have important influence on the bubbling performance of the microbubble generator. These important parameters are obtained by the traditional experimental method, which must waste much manpower, material, time and money. In this paper, the computer simulation analysis is used for the internal flow fields of the vortex microbubble generator. The structure parameters are selected for optimal design and the influences such as high cost, much time etc. of the experimental method are avoided.

\section{Introduction}

Microbubble generator in air flotation process plays a vital role, there is no suitable of the microbubble generator, the air flotation water treatment can not clean water [1]. And in order to design a good microbubble generator, the traditional method is to design, manufacture a prototype, experiment, and modify the design, manufacture a prototype again, then experiment, and then modify the design, ...... and finally a right result is got. These will undoubtedly spend a lot of manpower, material resources, time and money, and the design and manufacturing cycle is much length. Based on computational fluid dynamics and computer simulation technology, the paper studies the computer simulation method for analyzing the bubbling performance of the vortex microbubble generator, and basically determines the design parameters, reduces the experimental cost and improves the design efficiency. At the same time, it proves that the computer simulation and computational fluid dynamics analysis of the microbubble generator is useful and provides an important reference for the research and development of the same kind of fluid equipments.

\section{Working Principle of the Vortex Microbubble Generator}

The size, number and distribution of the microbubbles have a great influence on the water treatment. So it is very important for the bubbling properties of the microbubble generator.

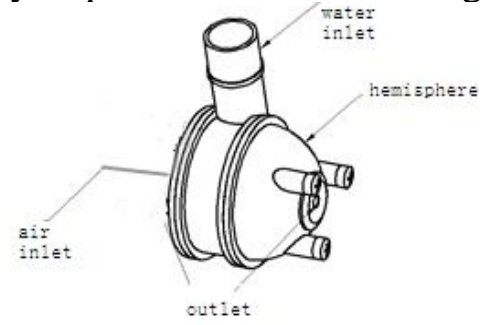

Fig. 1 Schematic Diagram of the Vortex Microbubble Generator

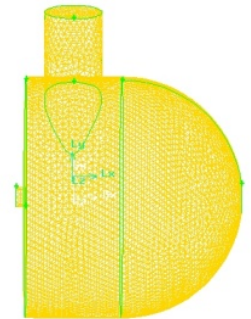

Fig. 2 The Mesh Partition Sketch of the Vortex Microbubble Generator 
Fig. 1 is the schematic diagram of self-sucking vortex microbubble generator [2]. The fluid with a certain initial velocity along the tangential direction from the inlet flows into the microbubble generator and generates swirling flow inside. According to the conservation of momentum moment and Bernoulli equation:

$$
u_{\partial} r=C
$$

$u_{\partial}$ is the fluid flow velocity in the tangential direction, $\mathrm{m} / \mathrm{s} ; \mathrm{r}$ is the radius of rotation of the fluid particle, $\mathrm{m}$; $\mathrm{C}$ is the constant of the moment momentum.

$$
p+\frac{1}{2} \rho v^{2}+\rho g h=c
$$

p is fluid pressure, MPa; $\rho$ is the density of fluid, $\mathrm{kg} / \mathrm{m}^{3}$; $\mathrm{v}$ is velocity, $\mathrm{m} / \mathrm{s}$; h is vertical height, $\mathrm{m}$; $\mathrm{g}$ is acceleration of gravity, $\mathrm{m} / \mathrm{s}^{2} ; \mathrm{C}$ is a constant, dimensionless.

In ideal conditions, if the energy loss of the fluid is not considered and the flow is always as the laminar flow, the flow from the tangential direction into the inside of the microbubble generator, the moment momentum keeps in a constant, as the point is more close to the center, its radius of rotation is less and the tangential velocity is larger. With the continuous variation of tangential velocity, it can be known from the Bernoulli equation, at this moment, the pressure energy will convert into kinetic energy, the point is more close to the center, the tangential velocity is larger and larger, the pressure will become smaller and smaller. A negative pressure zone will be generated near the middle axis zone of the microbubble generator. At the negative pressure, the air will be sucked into the vortex microbubble generator from the air suction nozzle (air inlet) and then go out from the mixture nozzle outlet due to different diameters of the inlet and the outlet, and the negative pressure values at both sides are also different, so that will produce in the middle axis of a pressure gradient. The sucked air will be mixed and broken into microbubbles and then sprayed out from the mixture outlet.

\section{Three Dimensional Modeling of the Vortex Microbubble Generator}

In this paper, the structure of the vortex microbubble generator is very simple, the 3D modeling can be designed by the software GAMBIT that belongs to the FLUENT [3]. First, to determine the coordinates of the contour model, by these coordinates generated contour curves, which scanning into the surface. Then, the real parts can be got, the entity components and the complete model are obtained through Boolean operation.

The Tet/Hybrid (tetrahedral / mixed) model is chosen as mesh partition [4]. The division model of the main mesh unit uses the tetrahedral mesh unit, and at same time in some irregular area and boundary positions the hexahedron and wedge meshes are used. This mesh partition method can produce detailed, tight and regular meshes, which can improve the simulation efficiency and reliability. According to the characteristics of the vortex microbubble generator, we will control the number of meshes around 200,000. Although more meshes can ensure the precision of simulation, the increasing meshes will increases the calculating time. After reaching a proper precision, less number of meshes will be better. Fig. 2 is the mesh partition sketch of $50 \mathrm{~mm}$ inner diameter of the vortex microbubble generator (the number of meshes is 176080).

\section{Simulation Parameters Setting of the Vortex Microbubble Generator}

(1) Simulation type setting: Because the shape of the vortex microbubble generator is asymmetrical and the calculation accuracy is general, a 3D model and single precision are selected.

(2) Simulation characteristics setting: The characteristics of simulation can be divided into Pressure Based Simulation and Density Based Simulation. The Pressure Based Simulation is mainly aimed at the solution of the incompressible flow in the low velocity, and the Density Based Simulation is mainly aimed at the solution of the compressible flow and high velocity. Although the 
fluid in the microbubble generator contains some air, the mainstream of the fluid is incompressible liquid (water), the velocity is not too high, it is appropriate to use the Pressure Based Simulation.

(3) Multiphase flow model setting: Because of the purpose of this paper is to study the air phase flow and the liquid phase flow, and in the microbubble generator the dirt in water usually is not easy to generate relative motion and in the colloidal form, based on simplifying a model, the dirt and impurities are negligible. The mixed multiphase flow model (mixture) is selected.

(4) Turbulence model setting: Reynolds Stress Model (RSM) is suitable for simulating the swirling flow field. It can be used for simulating the flow field of the vortex microbubble generator.

(5) Number of phases setting: Number of phases is set as two phases respectively water and air, and the properties of the two phases [5] are shown in Table 1.

Table 1 Material Properties

\begin{tabular}{|c|c|c|}
\hline Phase & Density $(\mathrm{kg} / \mathrm{m} 3)$ & Viscosity $(\mathrm{kg} / \mathrm{m} \cdot \mathrm{s})$ \\
\hline Water & 998 & 0.001003 \\
\hline Air & 1.225 & $1.7894 \times 10-5$ \\
\hline
\end{tabular}

(6) Boundary conditions setting: From the point of view of the structure of the vortex microbubble generator, the sewage inlet should be set as the entrance. VELOCITY-INLET expresses the initial velocity of sewage flowing into the microbubble generator. The value is set as $2 \mathrm{~m} / \mathrm{s}$. The computational fluid dynamics simulation is divided into two steps in this paper. In the first step the boundary conditions of the air suction inlet and mixture outlet at the same time are set up as the pressure outlets (PRESSURE-OUTLET), to determine the influence of the structure parameters on the internal performance of the vortex microbubble generator. In the second step MIXTURE model is set, the air is sucked at the negative pressure, and the internal flow fields are simulated by the structure parameters that are determined at the first step.

(7) Computation setting: For the air phase and the aqueous phase, the containing fraction, velocity components and turbulent components all of them use first-order discrete format, element node uses the first order upwind difference mode, the pressure velocity coupling uses the SIMPLEC algorithm.

(8) Others setting: Since it is a three-dimensional simulation, it can take into account the effect of gravity, the gravity acceleration is $9.8 \mathrm{~m} / \mathrm{s}^{2}$. Time property is set as constant, velocity mode takes absolute velocity.

(9) Convergence judgment setting: According to the general rule of convergence, the calculation is convergence as the residual value of each solution is less than 0.001 . But only observing the residual value is not enough and possible to be error, it is needed to check if the entire mass and momentum of the system are balance and the energy is conservation. The corresponding error values should be in the $0.1 \%$ if they are in a balance. If above requirements are achieved, it can determine that the calculation is convergence.

\section{Main Parameters of the Characteristics of the Flow Field}

The main purpose of the simulation of the vortex self-sucking microbubble generator is to optimally design the structure parameters for getting proper requirements of the flow field characteristics and good bubbling property. The main parameters include:

Flow static pressure P: The hydrostatic pressure of the internal flow field distribution has an important influence for the microbubble generator performance. In the vortex self-sucking microbubble generator at the air inlet it should have a larger negative pressure, in this way it can be more conducive for the air suction; At the internal wall surface, the pressure should be small. A small static pressure needs low requirement on the compressive and wearable properties of the material, and could reduce the machining cost, at the same time, reduce the wear of the wall. The distribution of the static pressure should have a good pressure drop gradient.

Turbulent kinetic energy T: The internal air will be stirred and broken into the tiny air bubbles by the role of the turbulent kinetic energy. Therefore, the greater of the internal turbulent kinetic energy of the microbubble generator should be better and the quality of the bubbles will be better, too. 
Outlet velocity V: The higher velocity at the outlet means the greater energy of the bubble has and bubbles with larger energy are more likely to flow out to collide with and adhere to the dirt in the water. The effect of the air flotation will have a beneficial effect.

Computer Simulation Examples are as sown in the Fig.3 and Fig.4.

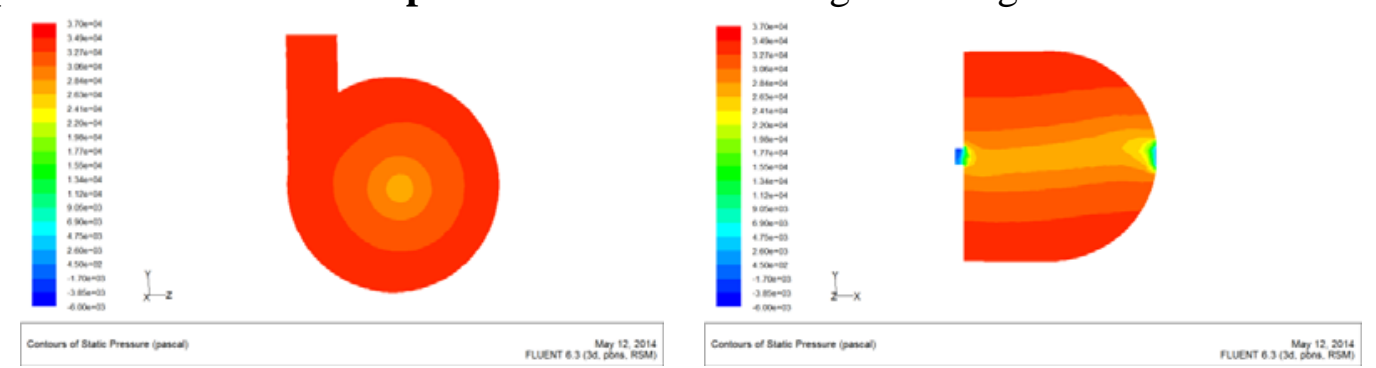

Fig. 3 The Static Pressure Distributions

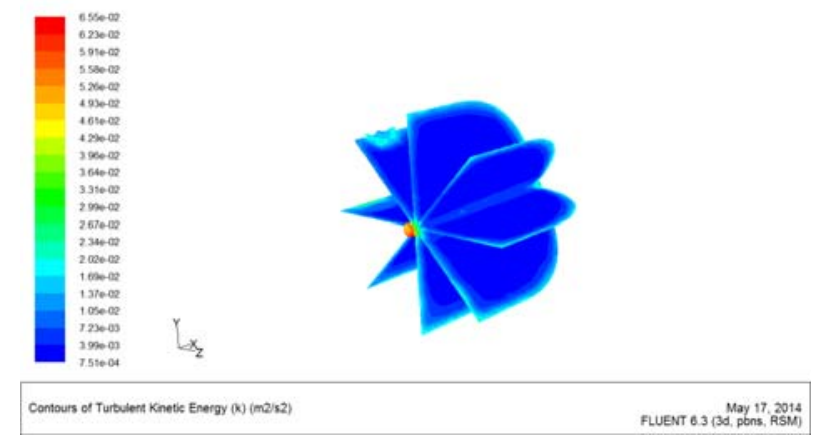

Fig. 4 Distributions of Turbulent Kinetic Energies in the Different Cross Sections of Inner Chamber

\section{Conclusions}

This paper analyzed the working principle of the vortex microbubble generator and some noticeable problems of internal flow field simulation based on computational fluid dynamics. It also studied the computer simulation methods for analyzing the bubbling performance of the vortex microbubble generator, provided an important reference for the development of fluidic devices. The simulation can get important parameters of the vortex microbubble generator and overcome the disadvantages of the traditional experimental method and save much manpower, material, time and money.

\section{Acknowledgements}

This work was sponsored by China Natural Science Foundation Project (No. 51168020) and Yunnan Province Natural Science Foundation Project (No. 2013FZ024). We will thank here!

\section{References}

[1] Li Zhekun, Li Min, Qiang Yuanli. Study on microbubble generators for treatment of wastewaters. 5th International Conference on Responsive Manufacturing - Green - Manufacturing, ICRM 2010, Vol (2010), No 565, 488-493

[2] Deng Jiushuai, Li Zhekun, He Wei, Yu Guorui, Fan Yujin, Shi Wenli, He Banggui,. A vortex of microbubble generator. Patent number: 2014203772964, ( 2015.01.28)

[3] Zhang Kai. Fluent technology and application examples. Beijing: Tsinghua University press, ( 2010.9)

[4] Wang Fujun. Computational fluid dynamics analysis. Beijing: Tsinghua University press, (2004.9)

[5] Horst Stöcker (DE) work. Wu Xizhen, Li Zhuxia, Chen Shiping translating. Handbook of physics. Beijing: Peking University Press, ( 2004.1) 